\title{
Dilepton-tagged jets in relativistic nucleus-nucleus collisions: A case study
}

\author{
Dinesh K. Srivastave* and Charles Gale \\ Physics Department, McGill University, 3600 University Street, Montreal, H3A 2T8, Canada \\ T. C. Awes \\ Oak Ridge National Laboratory, Oak Ridge, Tennessee 37831-6372, USA
}

(Dated: November 1, 2018)

\begin{abstract}
We study the $A+B \rightarrow \ell^{+} \ell^{-}+$jet $+\mathrm{X}$ process in nucleus-nucleus collisions at relativistic energies. The dilepton as well as the jet will pass through the matter produced in such collisions. The recoiling dilepton will carry information about the kinematical features of the jet, and will thus prove to be a very effective tool in isolating in-medium effects such as energy-loss and fragmentation function modifications. We estimate the contributions due to correlated charm and bottom decay and we identify a window where they are small as compared to pairs from the NLO Drell-Yan process.
\end{abstract}

\section{INTRODUCTION}

The study of dilepton production through the DrellYan process [1], $h_{1}+h_{2} \rightarrow \ell^{+} \ell^{-}+X$ has remained a useful tool to sound out concepts about the parton model, QCD, and the structure functions of hadrons. If the initial state partons have no transverse momentum, the lowest-order process $q \bar{q} \rightarrow \gamma^{*} \rightarrow \ell^{+} \ell^{-}$produces a lepton pair with a net $q_{T}=0$. Experiments, however, show that the net transverse momentum of the dileptons produced by the Drell-Yan process are of the order $1 \mathrm{GeV}$ for a dilepton having a mass $M \sim 10 \mathrm{GeV}$ [2]. This fact outlines the importance of the NLO Drell-Yan contributions. Also, one could assign an intrinsic spread to the transverse momenta of the partons. This momentum can have many origins, and a confinement argument along those lines only accounts for $\left\langle q_{T}^{2}\right\rangle \sim(0.3 \mathrm{GeV})^{2}$ [3].

It is therefore clear that the dileptons acquire additional transverse momenta from production mechanisms beyond leading order in perturbation theory:

$$
q g \rightarrow q \gamma^{*} \quad \text { and } \quad q \bar{q} \rightarrow g \gamma^{*}
$$

These Compton and annihilation processes are analogous to the ones responsible for the production of real photons where the recoiling final state quark or gluon balances the $q_{T}$ of the dilepton. An important point [4] is that the dileptons thus produced are always accompanied by a recoiling quark or gluon. If the energy of the quark or the gluon is several tens of $\mathrm{GeV}$, it will lead to a jet of hadrons in a narrow cone around the leading hadron in the jet.

Now consider a collision of two heavy nuclei at relativistic energies, which could lead to the formation of a quark gluon plasma (QGP). The dilepton and the jet produced in the above process will pass through the plasma. The dileptons will not interact, while the jet will lose energy through collisions and radiation in the plasma and

\footnotetext{
*Permanent address: Variable Energy Cyclotron Centre, 1/AF
} Bidhan Nagar, Kolkata 700 064, India provide valuable information about these mechanisms in the medium. The dilepton can thus be used to tag the jet in the same manner that photon-tagged jet measurements have been suggested as a precise probe for the study of jet-quenching [5].

We recall that jet-quenching can manifest itself in various ways; there would be a suppression of hadrons having large transverse momenta, when compared to the results for $p p$ collisions, and the fragmentation function in $A A$ collision may be different from those seen in $e^{+} e^{-}$or $p p$ collisions, etc [5]. A precise determination of these effects will require knowledge of the energy of the parton produced in the hard collision which fragmented into the jet. Note that the inclusive transverse momentum distribution of the hadrons will not permit us to deduce the value for the parton $d E / d x$ unambiguously, as a hadron with a given $p_{T}^{\text {hadron }}$ can arise from the fragmentation of any parton having $p_{T}^{\text {parton }}=p_{T}^{\text {hadron }} / z$, where $p_{T}^{\text {parton }}$ is the momentum of the parton at the time of fragmentation, and $z(<1)$, is the fragmentation variable. Recall also that the vast amount of theoretical activity [6] in the last decade has yielded varying predictions for the dependence of $d E / d x$ on the energy of the parton and the properties of the medium. A precise knowledge of the energy of the progenitor of the jet of hadrons will provide the information necessary to settle these issues.

The very large background of hadrons in $A A$ collisions makes it very difficult to characterize the jet through canonical jet algorithms which typically prescribe energy measurements in some cone around the jet-axis. To minimize contributions from the large non-jet background, particles emitted beyond the cone or having an energy below some threshold are excluded in the jet measurement. This excluded energy, as well as the non-jet background energy, must be estimated and taken into account. Because of the difficulty of making these corrections, photon tagging of the jet is a valuable alternative or complement to the jet energy measurement, as the transverse momentum of the photon (real or virtual) is equal to that of the high energy parton produced in the collision.

In this work, a measurement of dilepton-tagged jets is suggested and given quantitative support. We argue that 


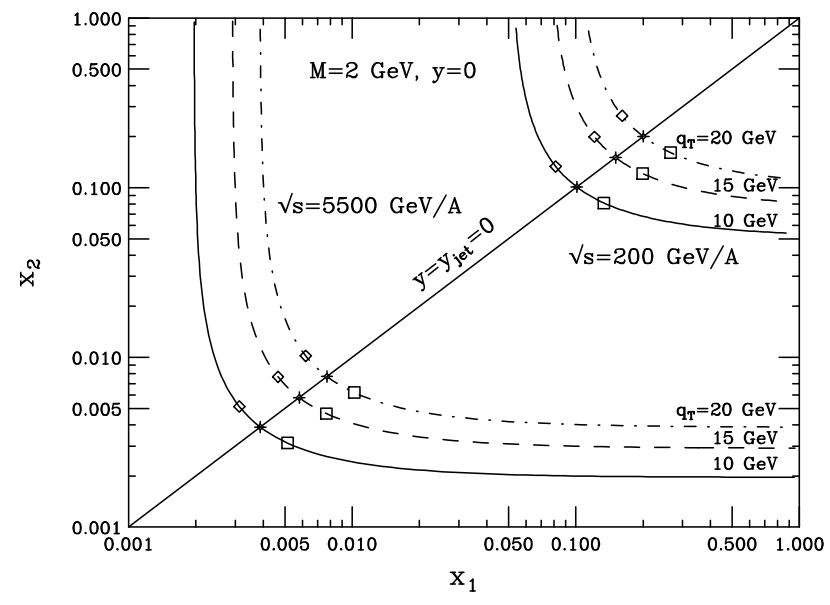

FIG. 1: The fractional momenta of the partons at RHIC and LHC energies relevant for the production of dileptons through the NLO Compton and annihilation processes. The symbols on the curves denote the points when $y=0$ and $y_{\text {jet }}=0.5$ (squares), 0.0 (crosses) and -0.5 (diamonds).

these measurements could have advantages over measurements of real photons, even though they are similar in philosophy. In measurements of real photons, photons from the radiative decays of mesons, predominantly $\pi^{0}$ 's, constitute a huge background which overwhelms the real photon yield except at very high transverse momenta. The real photon sample can be enhanced by rejecting photons for which an accompanying photon is measured with the two photons having a photon-pair mass which falls within the $\pi^{0}$ mass window. However, this method fails at low transverse momentum for central heavy ion collisions due to the need for a large acceptance in order to measure both photons with high probability, and due to the high $\pi^{0}$ multiplicity and the corresponding large number of combinatorial photon-pairs which results in the rejection of essentially all photons as possible decay photons. As the transverse momentum of the $\pi^{0}$ increases, such as when it is the leading hadron in the jet, the opening angle between the two decay photons decreases, making it possible to identify and reject the decay photons with high probability and with fewer false combinatorial possibilities. However, for photon measurements using calorimeters, at large transverse momentum the opening angle becomes so small that the symmetric decay photons will merge into a single shower cluster and appear as a single isolated photon, making it impossible to isolate them and discriminate between a single real photon and two overlapping photons from the decay of the $\pi^{0}$. This then leaves a narrow window of photon (jet) energies where the real photons can be identified and such studies may be conducted. Of course, quantitative limits will depend on actual detector characteristics of geometry and granularity, and on the particle multiplicity. Typical calorimeter geometries have $\pi^{0}$-photon shower merging limits as low as $20 \mathrm{GeV} / \mathrm{c}$.
Now consider the case of a dilepton recoiling against the jet. The $\ell^{+}$and $\ell^{-}$are easily separated for arbitrarily large momenta. There are no other sources of dileptons having large $q_{T}$ (see later). Furthermore, the mass of the lepton-pair $M$ gives an additional handle on the initial state scattering [5], as we can study dileptons which have the same transverse momentum (i.e. the recoiling jets have the same transverse momentum) but different masses. One pays the price of a low counting rate, but if the signal can be separated from the background and if luminosities are sufficient, we have a versatile tag.

To repeat, the dileptons produced through the process $q \bar{q} \rightarrow \ell^{+} \ell^{-}$will have only a modest $q_{T}$ resulting from the intrinsic transverse momentum of the partons. The correlated charm and bottom decays, which offer a huge contribution to the dilepton production, will also be governed by the same order of $q_{T}$ given from the intrinsic momenta of the partons, in lowest order. Even though the $c \bar{c}$ and the $b \bar{b}$ will have a vanishing $q_{T}$ in the lowest order, the random transverse momenta of the leptons in the semi-leptonic decay of the resulting $\mathrm{D}$ and $\mathrm{B}$ mesons could still result in a net $q_{T}$ for the lepton-pair. At the NLO, the heavy-quark pairs will have additional $q_{T}$, which would then get translated into the $q_{T}$ of the lepton-pair. One can see that the heavy quark will transfer only a part of its momentum to the lepton, (the semileptonic decays $\mathrm{B} \rightarrow \mathrm{D} \ell \nu$ and $\mathrm{D} \rightarrow \mathrm{K} \ell \nu$ have three-body final states) and thus there is hope that for large $q_{T}$ of the pair, the contribution of the heavy-quark decays may become smaller than the NLO Drell-Yan contribution. It is also likely that one may be able to suppress or account for these contributions explicitly if one can identify the vertex of the decaying $\mathrm{D}$ or $\mathrm{B}$ meson, or reject leptons within a region of jet-like activity. Moreover, if the charm and the bottom quarks lose energy due to collisions or radiations in the plasma [], their contribution will again be reduced [8], as the individual momenta of the quarks will be reduced, leading to a reduction in the net $q_{T}$ of the resulting lepton-pair.

These hopeful considerations are quantitatively tested in the following. Our goal here is to perform simple phenomenological estimates, and to identify kinematical domains appropriate to RHIC and to the LHC where our tag would shine through the background. Note in passing that the CMS experiment has considered the possibility of observing jets tagged by $\mathrm{W}$ and $\mathrm{Z}$ bosons, which are governed by the same criteria [9], and are thus open to similar vulnerabilities. See also [10].

\section{DILEPTON PRODUCTION}

At the outset, we add that the thermal radiation of dileptons from the QGP and the hot hadronic matter, a subject of considerable research [3] will not be important 11] in the domain of very large $q_{T}$ of interest here. The dileptons from the annihilation of jets passing through the QGP [12], could however have some contri- 
bution, at least at the LHC. We shall report on this in a future publication. In the following we consider only the Drell-Yan process and the correlated decay of heavy mesons.

\section{A. Drell-Yan : Lowest Order}

The cross section for the lowest-order Drell-Yan process is given by

$$
\frac{d \sigma}{d M^{2} d y}=\frac{4 \pi \alpha^{2}}{9 M^{4}} F\left(x_{1}, x_{2}, M^{2}\right)
$$

where

$$
\begin{aligned}
& F\left(x_{1}, x_{2}, M^{2}\right)=x_{1} x_{2} \sum_{i} \quad e_{i}^{2}\left[q_{i}^{A}\left(x_{1}, M^{2}\right) \bar{q}_{i}^{B}\left(x_{2}, M^{2}\right)\right. \\
& \left.+\bar{q}_{i}^{B}\left(x_{1}, M^{2}\right) q_{i}^{A}\left(x_{2}, M^{2}\right)\right]
\end{aligned}
$$

$q_{i}$ are quark structure functions, and the sum runs over the quark flavours. We further have,

$$
\begin{aligned}
& x_{1}=M e^{y} / \sqrt{s} \\
& x_{2}=M e^{-y} / \sqrt{s} .
\end{aligned}
$$

In the above $s$ is the square of the centre of mass energy per nucleon. The parton cross sections are scaled by the nuclear thickness function $T_{A B}(b=0)$ to obtain $d N / d M^{2} d y$ for the Drell-Yan production of dileptons in central (impact parameter $\mathrm{b}=0$ ) $\mathrm{A}+\mathrm{B}$ nuclear collisions. As observed in the introduction, if the partons do not have any intrinsic momentum then the net $q_{T}$ of these lepton pairs would be identically zero. The effect of including the intrinsic transverse momentum of the partons, to the LO Drell-Yan can be seen through the standard practice of folding-in a Gaussian distribution in $q_{T}$ with a width of $\sim 400 \mathrm{MeV}$. In the following we use the CTEQ4M parametrization [13] of the parton distributions.

\section{B. NLO Drell-Yan : Annihilation}

The NLO process for dilepton production has several interesting features relevant to the present study which we recall here. We reiterate that we are interested only in large $q_{T}$ processes, and thus the treatment discussed here is sufficient for this purpose 14]. A full treatment 15] which regularizes the behaviour of the cross section as $q_{T} \rightarrow 0$ is of course available. First consider the annihilation process, $q+\bar{q} \rightarrow g+\gamma^{*}$. The differential cross section for the production of a dilepton having mass $M$, transverse momentum $q_{T}$, rapidity $y$, with a jet associated at the rapidity $y_{\text {jet }}$ through the annihilation process is given by

$$
\frac{d \sigma^{\mathrm{A}}}{d M^{2} d^{2} q_{T} d y d y_{\mathrm{jet}}}=F\left(x_{1}, x_{2}, \mu_{F}\right) \frac{1}{\pi} \frac{d \widehat{\sigma}^{A}}{d M^{2} d \widehat{t}} .
$$

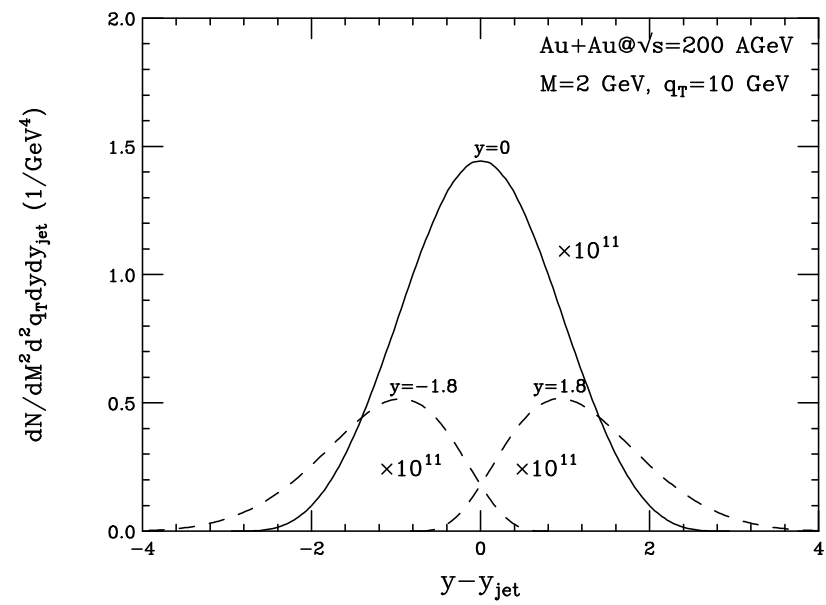

FIG. 2: Rapidity correlation of the dilepton and the recoiling jet at RHIC.

We have [4]

$$
\frac{d \widehat{\sigma}^{A}}{d M^{2} d \widehat{t}}=\frac{4}{9} \frac{2 \alpha^{2} \alpha_{s}\left(\mu_{R}\right)}{3 M^{2}} \frac{\left(\hat{t}-M^{2}\right)^{2}+\left(\hat{u}-M^{2}\right)^{2}}{\hat{s}^{2} \hat{t} \hat{u}},
$$

where

$$
\begin{aligned}
\hat{s}-M^{2} & =x_{1} x_{2} s-M^{2} \\
\hat{t}-M^{2} & =-\frac{1}{2} s \bar{x}_{T} x_{1} e^{-y} \\
\hat{u}-M^{2} & =-\frac{1}{2} s \bar{x}_{T} x_{2} e^{y} \\
\hat{t} \hat{u} & =\hat{s} q_{T}^{2}
\end{aligned}
$$

and

$$
\begin{aligned}
& x_{1}=\frac{1}{2} \bar{x}_{T} e^{y}+\frac{1}{2} x_{T} e^{y_{\mathrm{jet}}} \\
& x_{2}=\frac{1}{2} \bar{x}_{T} e^{-y}+\frac{1}{2} x_{T} e^{-y_{\mathrm{jet}}} .
\end{aligned}
$$

Above, any quantity under a caret (e.g. $\hat{\sigma}$ ) is associated with a parton-parton process. We set the renormalization and the factorization scales to $\mu_{R}=\mu_{F}=$ $M^{2}+q_{T}^{2}$, as appropriate for the study of the transverse momentum of the lepton-pairs. It is obvious that if one decides to choose $\mu_{F}=\mu_{R}=M^{2}$, the production of NLO Drell-Yan dileptons will change. We further have

$$
\bar{x}_{T}^{2}=x_{T}^{2}+4 \tau, x_{T}=\frac{2 q_{T}}{\sqrt{s}}, \tau=\frac{M^{2}}{s} .
$$

We also add that the momenta of the lepton pair $q$, the incoming partons $\left(p_{i}\right)$, and the recoiling parton $(k)$, in the nucleon-nucleon centre of mass system are given by:

$$
\begin{aligned}
p_{1} & =x_{1} \frac{1}{2} \sqrt{s}(1,0,0,+1), \\
p_{2} & =x_{2} \frac{1}{2} \sqrt{s}(1,0,0,-1), \\
q & =\left(q_{0}, \mathbf{q}_{T}, q_{L}\right), \\
k & =\left(k_{0},-\mathbf{q}_{T}, k_{L}\right) .
\end{aligned}
$$




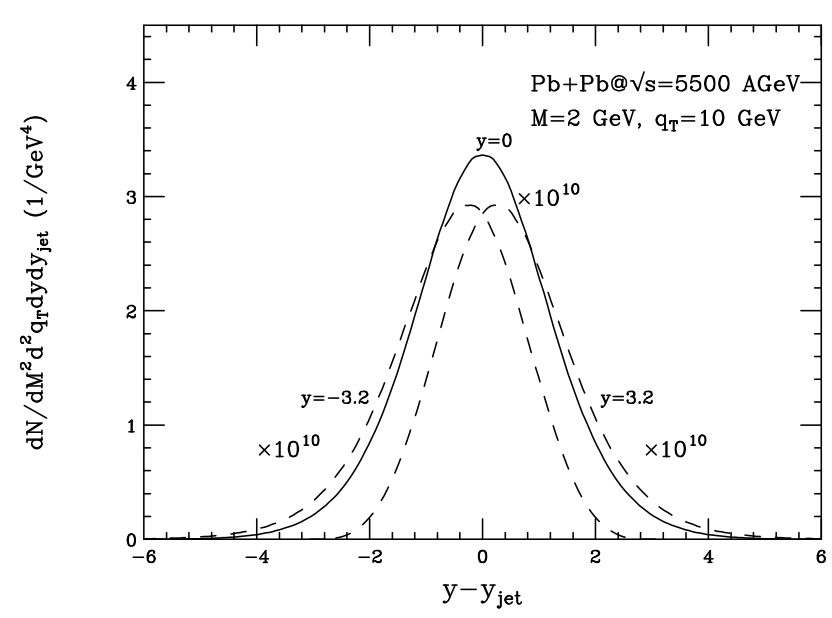

FIG. 3: Rapidity correlation of the dilepton and the recoiling jet at the LHC.

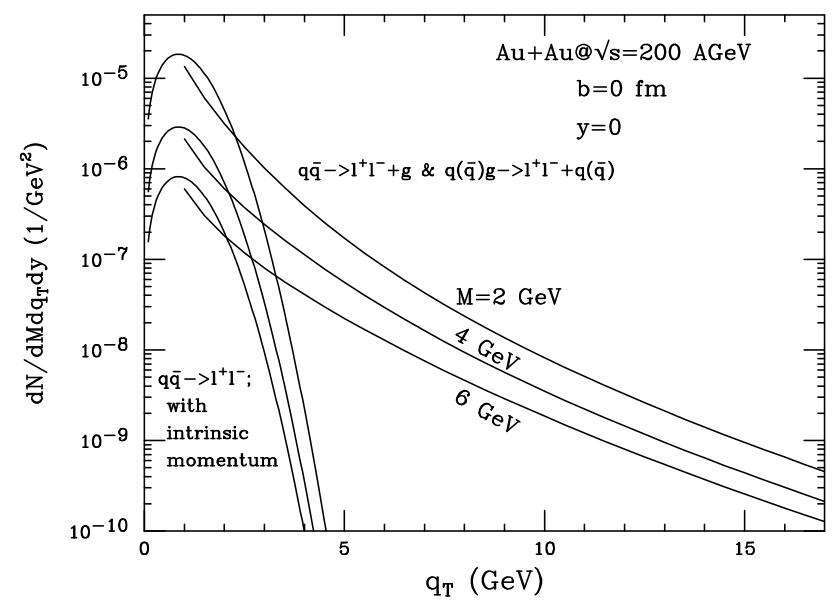

FIG. 4: Jet-rapidity integrated dilepton rates at RHIC for various masses of the pair.

\section{NLO Drell-Yan : Compton}

The expression for the Compton contribution to the Drell-Yan process at NLO is a little more involved. We have [4]

$$
\begin{aligned}
\frac{d \sigma^{\mathrm{C}}}{d M^{2} d^{2} q_{T} d y d y_{\mathrm{jet}}}= & F_{1}\left(x_{1}, x_{2}\right) \frac{1}{\pi} \frac{d \widehat{\sigma}^{C}(\hat{s}, \hat{u})}{d M^{2} d \widehat{u}}+ \\
& F_{2}\left(x_{1}, x_{2}\right) \frac{1}{\pi} \frac{d \widehat{\sigma}^{C}(\hat{s}, \hat{t})}{d M^{2} d \widehat{u}}
\end{aligned}
$$

where

$$
\begin{aligned}
& F_{1}\left(x_{1}, x_{2}\right)=x_{1} x_{2} g^{A}\left(x_{1}\right) \sum_{i} e_{i}^{2}\left[q_{i}^{B}\left(x_{2}\right)+\bar{q}_{i}^{B}\left(x_{2}\right)\right] \\
& F_{2}\left(x_{1}, x_{2}\right)=x_{1} x_{2} \sum_{i} e_{i}^{2}\left[q_{i}^{A}\left(x_{1}\right)+\bar{q}_{i}^{A}\left(x_{1}\right)\right] g^{B}\left(x_{2}\right)
\end{aligned}
$$

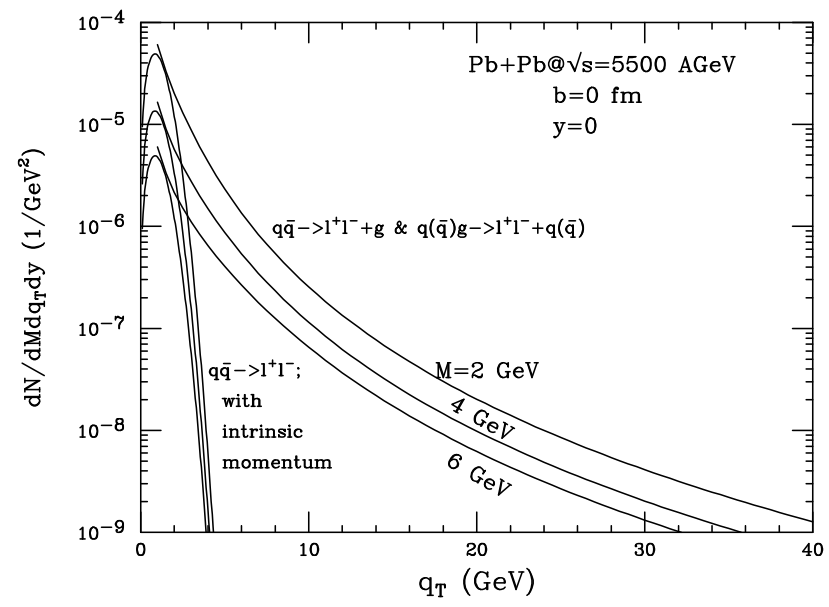

FIG. 5: Jet-rapidity integrated dilepton rates at the LHC for various masses of the pair.

$$
\frac{d \widehat{\sigma}^{C}(\hat{s}, \hat{u})}{d M^{2} d \widehat{u}}=\frac{1}{6} \frac{2 \alpha^{2} \alpha_{s}}{3 M^{2}} \frac{\left(\hat{s}-M^{2}\right)^{2}+\left(\hat{u}-M^{2}\right)^{2}}{\hat{s}^{3}(-\hat{u})}
$$

and

$$
\frac{d \widehat{\sigma}^{C}(\hat{s}, \hat{t})}{d M^{2} d \widehat{u}}=\frac{1}{6} \frac{2 \alpha^{2} \alpha_{s}}{3 M^{2}} \frac{\left(\hat{s}-M^{2}\right)^{2}+\left(\hat{t}-M^{2}\right)^{2}}{\hat{s}^{3}(-\hat{t})} .
$$

In the above, we have not explicitly shown the factorization and the renormalization scales, which are taken as $\mu_{F}=\mu_{R}=M^{2}+q_{T}^{2}$ as for the NLO annihilation term.

\section{Heavy-Quark Production}

We consider the production of heavy quarks at NLO. The lowest order contributions come from the processes $q \bar{q} \rightarrow Q \bar{Q}, g g \rightarrow Q \bar{Q}$. The NLO terms originate from $q \bar{q} \rightarrow Q \bar{Q} g, g q(\bar{q}) \rightarrow Q \bar{Q} q(\bar{q})$, and $g g \rightarrow Q \bar{Q} g$. The general expression for the production can be written as

$d \sigma=\sum_{i, j} \int d x_{1} d x_{2} d \widehat{\sigma}_{i j}\left(\hat{s}, M_{Q}^{2}, Q^{2}\right) f_{i}^{A}\left(x_{1}, Q^{2}\right) f_{j}^{B}\left(x_{2}, Q^{2}\right)$

where $f_{i}^{A}$ are the distribution functions for the partons in the nucleon in the nucleus $\mathrm{A}, x_{i}$ are the fractional momenta of the incoming partons, and $\hat{s}=x_{1} x_{2} s$ is the parton-parton centre of mass energy. The parton crosssection $\widehat{\sigma}_{i, j}\left(\hat{s}, M_{Q}^{2}, Q^{2}\right)$ can be written as:

$$
\widehat{\sigma}_{i, j}\left(\hat{s}, M_{Q}^{2}, Q^{2}\right)=\frac{\alpha_{s}^{2}\left(Q^{2}\right)}{M_{Q}^{2}} f_{i, j}\left(\rho, \frac{Q^{2}}{M_{Q}^{2}}\right),
$$

where

$$
\rho=\frac{4 M_{Q}^{2}}{x_{1} x_{2} s}
$$


and

$$
\begin{aligned}
f_{i, j}\left(\rho, Q^{2} / M_{Q}^{2}\right)= & f_{i, j}^{(0)}(\rho)+4 \pi \alpha_{s}\left(Q^{2}\right)\left[f_{i, j}^{(1)}(\rho)+\right. \\
& \left.\bar{f}_{i, j}^{(1)}(\rho) \ln \left(Q^{2} / M_{Q}^{2}\right)\right]
\end{aligned}
$$

are taken from Ref. [16]. We have used the factorization and the renormalization scales as $Q^{2}=M_{Q}^{2}+<p_{T}^{2}>$, where $\left\langle p_{T}^{2}\right\rangle$ is the average of the transverse momentum of the produced quark and anti-quark. The correlated semi-leptonic decay of the heavy (D or B) mesons is then estimated by fragmentation of the heavy-quarks to produce the meson and then estimating its decay in the quark model [17]. The branching ratios to leptons for the decay of the D and B mesons are taken as $12 \%$ and $10 \%$ 11], respectively. Even though more sophisticated approaches exist [18], that outlined above is sufficient for our purpose, as we only wish to know where these contributions are small. We have verified that our results are quantitatively similar to those obtained from PYTHIA [19] and also by Gavin et al. 11], where parametrizations of the measured lepton spectra from semi-leptonic decay of $\mathrm{B}$ and $\mathrm{D}$ mesons are used to generate the lepton momenta. The total cross sections for heavy $q \bar{q}$ we thus obtain are shown in Table【 These values do depend on the choice of structure functions and scales [20].

\begin{tabular}{c|c|c|c|c}
\hline \hline$\sqrt{s}(\mathrm{GeV})$ & $\sigma_{\mathrm{c \overline {c }}}^{\mathrm{LO}}$ & $\sigma_{\mathrm{cc}}^{\mathrm{NLO}}$ & $\sigma_{\mathrm{bb}}^{\mathrm{LO}}$ & $\sigma_{\mathrm{bb}}^{\mathrm{NLO}}$ \\
\hline 200 & 59.3 & 145.9 & 0.84 & 1.67 \\
5500 & 1054 & 3362 & 79.7 & 174.4 \\
\hline \hline
\end{tabular}

TABLE I: Total cross sections for heavy $q \bar{q}$ production in pp collisions. All values are in $\mu \mathrm{b}$.

Finally, the momentum lost by the heavy quarks during fragmentation is taken to be negligible 21].

\section{RESULTS}

As a first step we explore the range of fractional momenta of the partons (Eq. 8) which are relevant for our purpose (see Fig. 1). The diagonal provides the values for the situation when the rapidity of the dilepton $(y)$ as well as that of the recoiling jet $\left(y_{\mathrm{jet}}\right)$ is zero. The squares denote the point when the $y_{\text {jet }}=+0.5$, while the point $y_{\text {jet }}=-0.5$ is indicated by the diamonds, when the dilepton $y=0$. Thus large $M$ and very large $q_{T}$ of the lepton pair do not sample very small values of $x$, and the nuclear modification of the parton distribution functions will hence not play a large role.

Next we study the rapidity correlation between the dilepton and the jet. We see (Figs. 2 and 3) that there is a strong positive correlation between the rapidity of the dilepton and the recoiling jet; i.e. when the dilepton has a positive rapidity the jet also has a positive rapidity, and

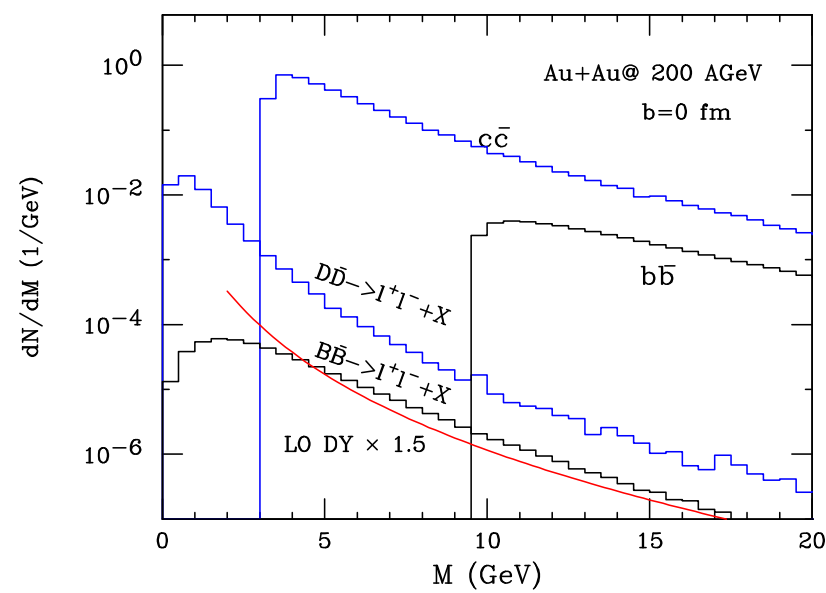

FIG. 6: Mass distribution of heavy quark pairs and the dileptons from their correlated decay at RHIC energies. The LO Drell-Yan contribution is also shown.

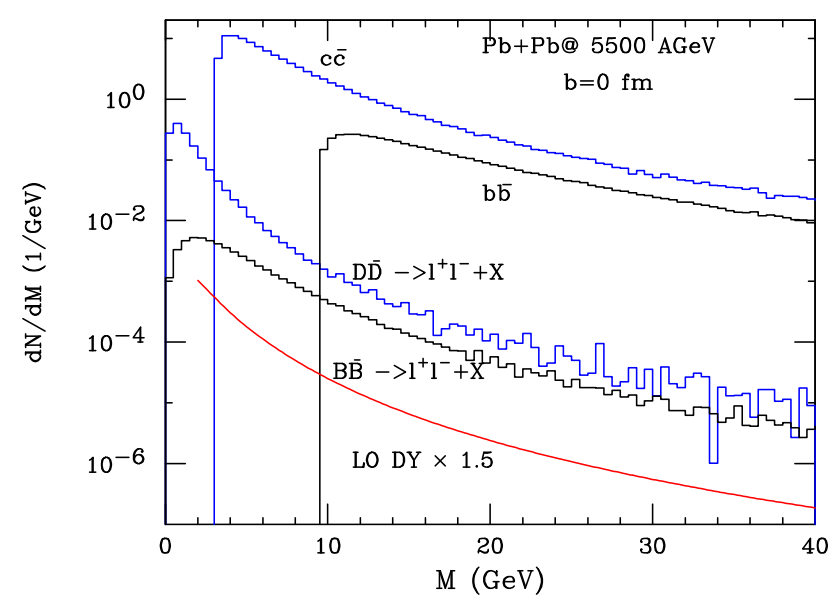

FIG. 7: Same as Fig 6 for the LHC

the correlation has a width of about 2 units of rapidity. This aspect has been discussed in detail by Kajantie et al. [4] and has been attributed to the dominance of the Compton term through the $1 / \widehat{s}$ term in the scattering cross section. Note that the finite $y$ values are chosen as the mid-points of the forward/backward rapidity coverage of PHENIX at RHIC, and they fall within those for the ALICE and CMS experiments at the LHC.

We now study the jet-rapidity integrated dilepton rates at RHIC (Fig. 4) and at the LHC (Fig. 5) due to the NLO processes discussed above. We have also given the results for the LO Drell-Yan contribution, supplemented with a modest intrinsic $k_{T}$ for the partons. As expected, the NLO processes dominate as the transverse momentum of the pair (which is also the transverse momentum of the recoiling jet) increases. The NLO contributions to the Drell-Yan and also the heavy-quark production (see below) have been calculated ignoring the intrinsic momenta of the partons. Including it will increase the $\left\langle q_{T}>\right.$ of 

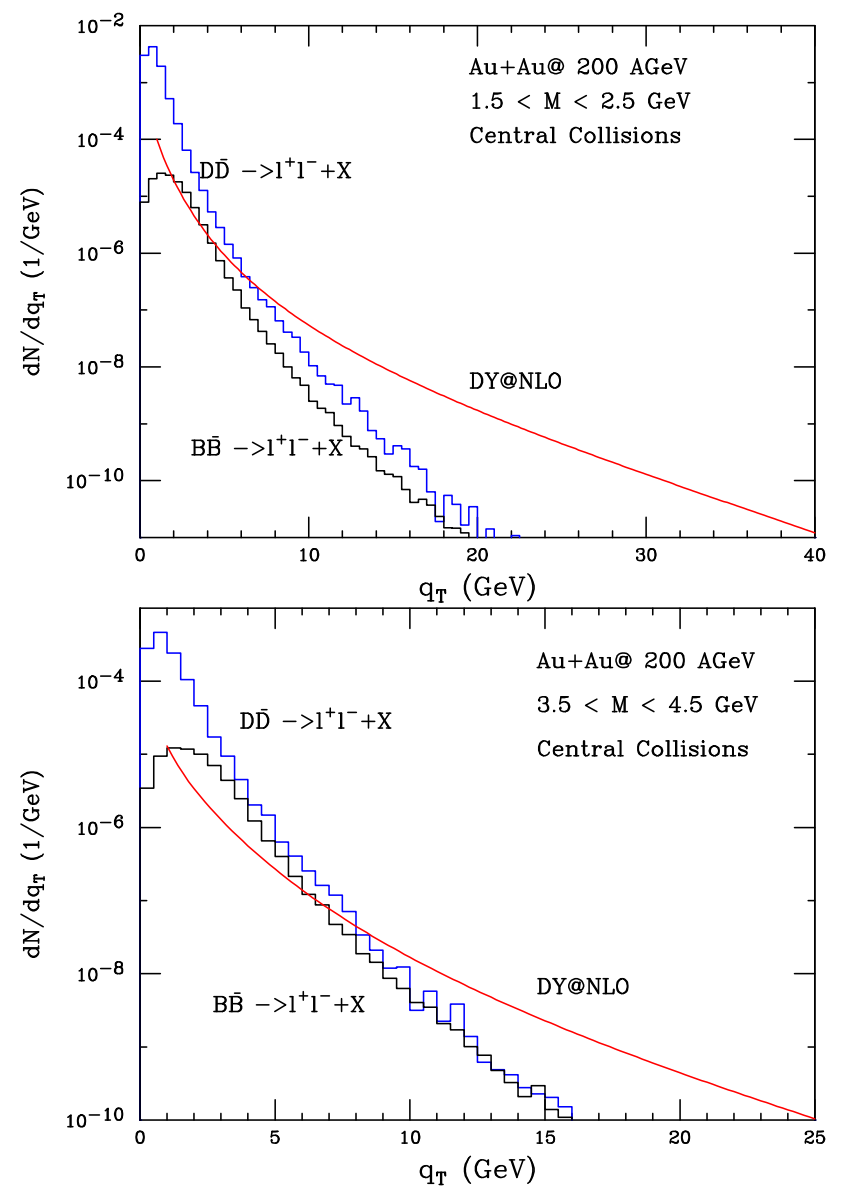

FIG. 8: Rate of dilepton production with pair mass $1.5<$ $M<2.5 \mathrm{GeV}$ (upper panel) and $3.5<M<4.5 \mathrm{GeV}$ (lower panel) as a function of pair transverse momentum at RHIC energies.

the resulting lepton-pair and may enhance the yield by 50-100\% depending on the value of the intrinsic momentum.

The semi-leptonic decay of $\mathrm{D}$ and $\mathrm{B}$ mesons gives a large contribution to the dilepton production 11]. We show the results of our calculations, which extend up to large lepton pair masses. The LO Drell-Yan contribution has been scaled by a factor of 1.5 to account for the NLO terms as our exploratory calculations will be inadequate at low $q_{T}$, and a momentum integral has to be performed in order to obtain the mass distributions.

Figs. [6] and 7] suggest that it may be very difficult to observe the dileptons recoiling against a jet in the background of the correlated decay of charm and bottom. This indeed is the case for dileptons radiated from thermal sources [11]. However, this conclusion changes when the $q_{T}$ distribution of the dileptons is studied for large pair masses, as shown in Fig. 8 for mass windows of 2 and $4 \mathrm{GeV}$ at RHIC energies. We find that dileptons originating from the NLO Drell-Yan process dominate at large $q_{T}$, and with anticipated total sampled event sizes
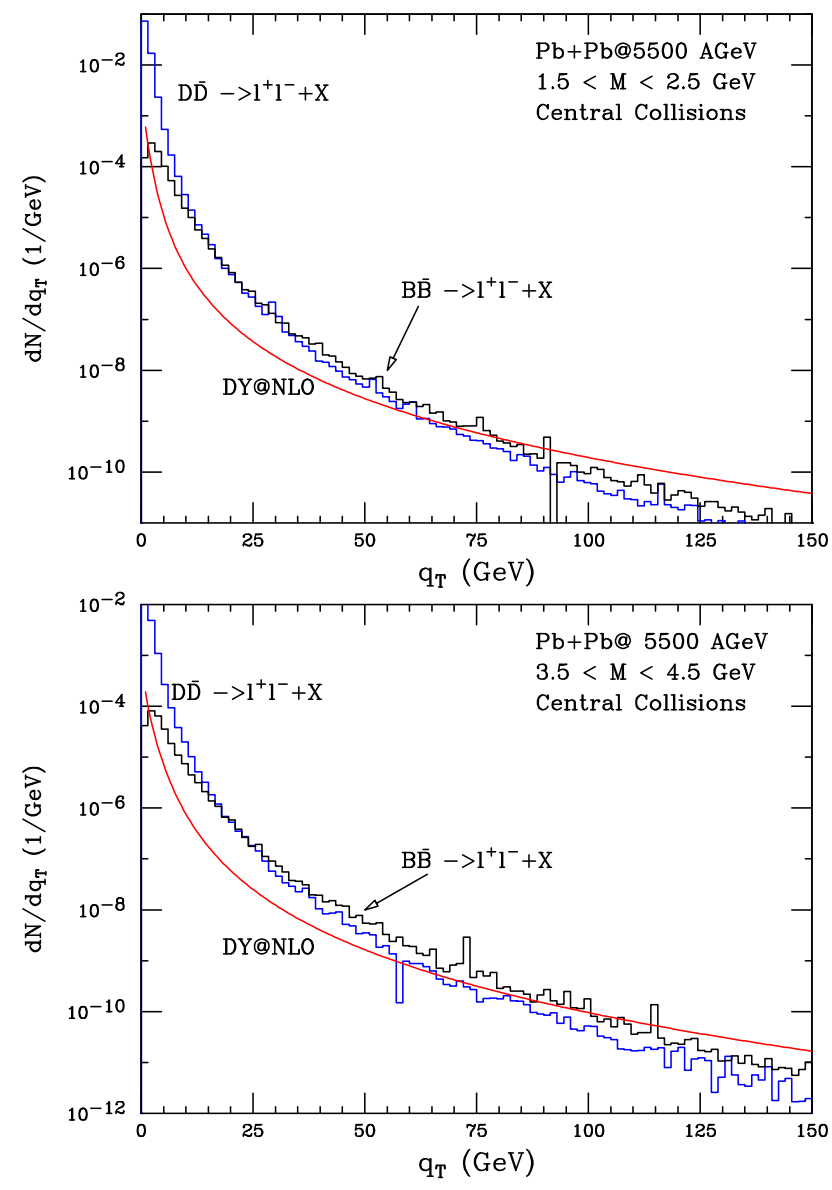

FIG. 9: Same as Fig 8 for the LHC.

of $\sim 10^{10}$ events, open a window for a clean measurement of dilepton-tagged-jets. This window shifts to higher $q_{T}$ at the LHC (Fig 9), and identification becomes more difficult than at RHIC. The situation may improve if the heavy-quarks lose energy in the medium, degrading the $\mathrm{D}$ and $\mathrm{B}$ meson decay contributions to lower dilepton $q_{T}$. Furthermore, the background can be reduced if the contributions of the heavy-meson decays can be isolated and rejected, such as by determination of the decay vertices, or by jet-like isolation cuts.

\section{CONCLUSIONS}

In brief, we have estimated the production of jets recoiling against a dilepton produced in the Drell-Yan process at NLO in relativistic heavy ion collisions at RHIC and LHC energies. The dileptons could be used to tag the jet and give the energy of the progenitor parton, which would provide precise information with which to determine the rate of energy loss of partons during their passage through quark gluon plasma. The background from the correlated decay of charm and bottom mesons, which plagues the identification of thermal dileptons [1], 
is found to be unimportant for large jet (or dilepton) transverse momenta, that is, in the kinematical region of interest for jet-quenching studies. Even though the counting rates will be small, the anticipated event samples suggest that dilepton jet-tagging is feasible. This method could thus prove useful in the resolution of several issues of fundamental importance in the physics of high energy partons traversing a plasma of quarks and gluons.

\section{Acknowledgments}

We thank M. Mangano for providing us with his code for heavy-quark production [16] and for many help- ful communications. This work was supported by the Natural Sciences and Engineering Research Council of Canada. ORNL is managed by UT-Battelle, LLC, for the U.S. Department of Energy under contract DE-AC0500OR22725.
[1] S. D. Drell and T. M. Yan, Phys. Rev. Lett. 25, 316 (1970).

[2] Richard D. Field, Applications of Perturbative QCD (Addison-Wesley, Redwood City, 1989).

[3] C. Gale, Nucl. Phys. A 698, 143c (2002).

[4] K. Kajantie, J. Lindfors, and R. Raitio, Nucl. Phys. B 144, 422 (1978).

[5] X. N. Wang, Z. Huang, and I. Sarcevic, Phys. Rev. Lett. 77, 231 (1996), X. N. Wang and Z. Huang, Phys. Rev. C 55, 3047 (1997).

[6] M. Gyulassy, P. Levai, and I. Vitev, Phys. Rev. D 66, 014005 (2002); R. Baier, D. Schiff, and B. G. Zakharov, Ann. Rev. Nucl. Part. Sci. 50, 37 (2000); R. Baier, Yu. L. Dokshiter, A. H. Mueller, and D. Schiff, Phys. Rev. C 58, 1706 (1908); U. A. Weidemann, Nucl. Phys. B 588, 303 (2000); B 582, 409 (2000); and references therein.

[7] B. Svetitsky, Phys. Rev. D 37, 2484 (1988); M. G. Mustafa, D. Pal, and D. K. Srivastava, Phys. Rev. C 57, 889 (1998); Erratum Phys. Rev. C 57, 3499 (1998); M. G. Mustafa, D. Pal, D. K. Srivastava, and M. Thoma, Phys. Lett. B 428, 234 (1998); see also, Y. L. Dokshitzer and D. E. Kharzeev, Phys. Lett. B 519, 199 (2001).

[8] E. V. Shuryak, Phys. Rev. C 52, 961 (1997); K. Gallmeister, B. Kämpfer, and O. P. Pavlenko, Phys. Rev. C 57, 3276 (1998); Z. Lin, R. Vogt, and X. N. Wang, Phys. Rev. C 57, 899 (1998).
[9] G.Baur, et al., CMS NOTE 2000/060.

[10] V. Kartvelishvili, R. Kvatadze, and R. Shanidze, Phys. Lett. B 356, 589 (1995).

[11] S. Gavin, P. L. McGaughey, P. V. Ruuskanen, and R. Vogt, Phys. Rev. C 54, 2606 (1996).

[12] D. K. Srivastava, C. Gale, and R. J. Fries, nucl-th/02090063.

[13] H. L. Lai et al. [CTEQ Collaboration], Eur. Phys. J. C 12, 375 (2000)

[14] Edmond L. Berger, Lionel E. Gordon, and Michael Klasen, Phys. Rev. D 58, 074012 (1998).

[15] P. Arnold and R. Kaufmann, Nucl. Phy. B 349, 381 (1991)

[16] M. L. Mangano, P. Nason, and G. Ridolfi, Nucl. Phys. B 373, 295 (1992).

[17] G. Altarelli, N. Cabibo, G. Carbo, L. Maiani, and G. Martinelli, Nucl. Phys. B 208, 365 (1982); N. Cabibo, G. Carbo, and L. Maiani, Nucl. Phys. B 155, 93 (1979).

[18] N. Isgur, D. Scora, B. Grinstein, and M. B. Wise, Phys. Rev. D 39, 799 (1989).

[19] T. Sjöstrand, L. Lonnblad, and S. Mrenna, hep$\mathrm{ph} / 018264$.

[20] R. V. Gavai et al., Int. J. Mod. Phys. A10, 2999 (1995).

[21] R. Vogt, S. J. Brodsky, and P. Hoyer, Nucl. Phys. B383, 643 (1992). 